\title{
An Efficient Geometric Parametrization Technique for the Continuation Power Flow through the Tangent Predictor ${ }^{1}$
}

A. BONINI NETO ${ }^{2}$, D.A. ALVES ${ }^{3}$, Electrical Engineering Department, FEIS, São Paulo State University (UNESP), 15385-000 Ilha Solteira, SP, Brazil.

\begin{abstract}
This paper presents a new parameterization scheme to the continuation power flow that allows the complete tracing of $\mathrm{P}-\mathrm{V}$ curves and the computation of the maximum loading point of power system, without ill-conditioning problems of the Jacobian matrix and without the exchange of parameter. The objective is to present the technique in a clear and didactic way.
\end{abstract}

Keywords. Load Flow, Multiple solutions, Maximum Loading Point.

\section{Introduction}

An important task of voltage stability analysis is the computation of the system's maximum loading point (MLP). This is important for the knowledge of voltage stability margin and also for modal analysis studies, which uses the MLP to assess the critical eigenvectors and eigenvalues of the Jacobian matrix. Usually, the MLP is obtained by successive power flow solutions through manual scaling of the loading level of the system. This procedure is repeated until the power flow method (PF) becomes divergent. For practical purposes this point is considered as the MLP [7]. The MLP is associated to a physical limitation of the power network for a particular configuration, load condition, load increase direction etc. and, should not be based on a mathematical limitation of the numerical method. As shown in [3], the point where PF calculations fail to converge may vary, depending on which method is used in the calculation. It was shown in [3] that different MLPs were obtained with the conventional Newton and fast decoupled methods. It is well known that the convergence problems of conventional Newton methods during the computation of the MLP are consequences of numerical difficulties associated with the singularity of the Jacobian matrix. This singularity occur in systems with constant PQ loads because, in this case, the gradual load increment will lead to a saddle-node bifurcation point, which corresponds to the MLP. Therefore, the divergence will occur even if double-precision computation and anti-divergence algorithms are used. Thus, this class of methods is not adequate for tracing complete $\mathrm{P}-\mathrm{V}$ curves, being restricted

\footnotetext{
${ }^{1} \mathrm{CNPq}$ and FAPESP.

2 alfredoneto@aluno.feis.unesp.br

33dalves@dee.feis.unesp.br
} 
to the upper portion of the curves until the vicinity of the MLP. In general, the ability of the power flow method to find a solution will depend on the existence of the solution or multiple solutions, the presence of singularity, the solution method, and the initial conditions.

The application of continuation methods for voltage stability analysis overcome the above mentioned difficulties by adding parameterized equations $[1,2,3,4,5,7$, 10]. The load flow equations are reformulated in order to eliminate the singularity of the Jacobian matrix $(J)$, and consequently the numerical problems that occur in its vicinity at the MLP. A critical point for a successful continuation method is the parameterization step, which has been the subject of many investigations reported in the literature. The most used parameterization techniques can be classified as local parameterization [1] and geometrical parameterization [4, 5]. The local techniques consist of changing the parameter near the MLP in a way that the singularity of $J$ disappears. Geometrical techniques add equations of lines or arclengths as parameters. For instance, a line equation perpendicular to the tangent vector was used in [6]. It provided good results but a very precise step size control around the MLP was necessary [4]. In [5] an arc-length equation was proposed as parameter. This technique allows the complete tracing of $\mathrm{P}-\mathrm{V}$ curves with no need of changing the parameter during the process. However, as it includes a non-linear equation at the predictor step, the resulting set of equations requires the application of special methods to be successfully solved, which can be very time-consuming [5]. An important characteristic of continuation methods is that they can provide valuable information regarding the geometry of the solution space of the power flow equations, which is useful not only from a didactic point of view, since it facilitates the understanding of the problem, but it can also aid the development of new strategies for the elimination of numerical problems related to the solution methods, the computation of multiple solutions and obtaining of voltage stability indices [8].

The parameterization techniques based on physical parameters simplify the mathematical definition and understanding of the methods [1, 2]. However, the major part of continuation methods found in the literature use complex parameterization techniques with purely geometric interpretation $[4,5]$. The latter are considered more robust and therefore, more appropriate than those based on physical parameters [7].

This paper presents a new geometric parameterization scheme that allows the complete tracing of the $\mathrm{P}-\mathrm{V}$ curves without ill-conditioning problems of the matrix $J$. The Jacobian matrix singularity is avoided by the addition of a line equation, which passes through a point in the plane determined by variables loading factor and bus voltage magnitude. The use of this technique enlarges the group of voltage variables that can be used to tracing of $\mathrm{P}-\mathrm{V}$ curves.

\section{Continuation Power Flow (CPF)}

The definition of a CPF method starts with the general power flow equations for a given system, which can be written as

$$
G(V, \theta, \lambda)=0
$$


where $V$ is the vector of voltage magnitudes and $\theta$ is the vector of voltage phase angles. The symbol $\lambda$ is a loading factor used to scale up the loading and generation level and $G$ is a vector of equations representing the real and reactive power balance of system buses. These equations can be written as

$$
\begin{aligned}
& {\left[P_{\text {gen }}(\lambda)-P_{\text {load }}(\lambda)\right]-P(\theta, V)=0} \\
& {\left[Q_{\text {gen }}-Q_{\text {load }}(\lambda)\right]-Q(\theta, V)=0}
\end{aligned}
$$

here $P_{\text {load }}(\lambda)=\lambda k_{\text {pl }} P_{\text {load }}^{s p}, Q_{\text {load }}(\lambda)=\lambda k_{q l} Q_{\text {load }}^{s p}$, and $P_{\text {gen }}(\lambda)=\lambda k_{\text {gg }} P_{\text {gen }}^{s p}, P_{\text {load }}^{s p}$, $Q_{\text {load }}^{s p}$, and $P_{\text {gen }}^{s p}$ are specified values at base case $(\lambda=1)$ for real and reactive power of $P Q$ buses and real power for $P V$ buses, respectively. The symbols $k_{p g}, k_{p l}$ and $k_{q l}$ are pre-specified parameters used to adjust a specific loading scenario, describing the rate of changing of real power $\left(P_{\text {gen }}\right)$ for generation buses $(P V$ buses $)$, and real $(P)$ and reactive $(Q)$ power for load buses ( $P Q$ buses). Therefore, it is possible to apply an individual loading variation, for a single bus or for a selected group of buses, considering different power factors (from the base case values) for the buses. If system security is evaluated through the maintenance of a minimum voltage stability margin, a constant power load representation will result in a more secure operational system condition [9]. Once a loading and generation pattern is defined, (2.2) can be solved by using a conventional PF method (e.g. Newton) to compute solutions for various loading conditions. This is done by increasing $\lambda$ gradually from 1 (base case) up to a value for which a solution can no longer be found ( $P F$ calculations diverge). In this case $V$ and $\theta$ are dependent variables, while $P_{\text {gen }}, P_{\text {load }}, Q_{\text {load }}$ and $\lambda$ are independent variables, i.e., $\lambda$ is treated as a parameter in the Newton iterative process. On the other hand, in continuation method procedures in general, $\lambda$ is considered as a dependent variable and then, changed automatically. In this case, equation (2.2), which dimension is $n=2 n_{P Q}+n_{P V}\left(n_{P Q}\right.$ and $n_{P V}$ correspond to the number of $P Q$ and $P V$ buses, respectively) now has $n+1$ unknowns, and an additional equation is needed. The difference among the continuation methods is in the way this new variable is handled and how singularity of the Jacobian matrix is avoided. Among the many methods described in the literature $[1,2,7]$, the most widely used consist of four basic elements: a parameterization procedure, a predictor step, a step size control, and a corrector step.

\section{Proposed Continuation Power Flow}

In order to eliminate the drawback of continuation methods, the methodology proposed in this paper adds a new equation to (2.1). The idea is to use a line equation (see Figure 1) that passes through any chosen point $\left(\lambda^{0} ; V^{0}\right)$ at the plane defined by variables loading factor $(\lambda)$ and buses voltages magnitude $(V)$ :

$$
\begin{aligned}
& G(\theta, V, \lambda)=0 \\
& W(\theta, V, \lambda, \alpha)=\alpha\left(\lambda-\lambda^{0}\right)-\left(V_{k}-V_{k}^{0}\right)=0
\end{aligned}
$$

where the parameter $\alpha$ is the angular coefficient of the line. As a new equation is added, $\lambda$ can be treated as dependent variable and $\alpha$ is considered as continuation parameter. 


\subsection{Parameterization and Predictor Step}

Starting from a solved base case $\left(\theta^{1}, V^{1}\right.$ and $\left.\lambda^{1}\right)$ plus the initial chosen point $\left(\lambda^{0} ; V^{0}\right)$, a value for $\alpha$ is computed by

$$
\alpha^{1}=\frac{V_{k}^{1}-V_{k}^{0}}{\lambda^{1}-\lambda^{0}} .
$$

Once $\alpha$ computed, the proposed continuation power flow method (PCPF) can be used to trace the P-V curve and to compute the MLP by applying the predictor step to find an estimate for the next solution. The most common prediction techniques estimate solutions on the tangent or secant lines computed from converged points $[1,5,10]$.

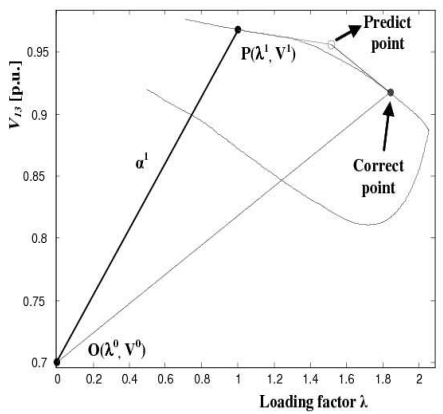

Figure 1: Tangent predictor with $\alpha$ as parameter.

In the tangent method, the estimate of the next solution can be found by taking an appropriately sized step in a direction tangent to the solution path at the current solution. The tangent vector is computed by taking the derivative of (3.1)

$$
\left[\begin{array}{ccc}
-J & -G_{\lambda} & 0 \\
\frac{\partial W}{\partial x} & -\alpha & \lambda-\lambda^{0} \\
0 & 0 & 1
\end{array}\right]\left[\begin{array}{l}
d x \\
d \lambda \\
d \alpha
\end{array}\right]=J_{m} t=\left[\begin{array}{c}
0 \\
0 \\
\pm 1
\end{array}\right]
$$

where $x=\left[\begin{array}{ll}\theta^{T} & V^{T}\end{array}\right]^{T}, G_{\lambda}$ corresponds to the partial derivatives of $G$ with respect to $\lambda, J$ is the Jacobian matrix of the conventional PF, $J_{m}$ is the Jacobian matrix modified due to the increase in the number of rows and columns and $t$ the tangent vector. The sign choice $(+1$ or -1$)$ will depend on how variable $\alpha$ will be changing as the solution path is being traced. Plus if it is increasing, and minus if it is decreasing. After solving (3.3) for the tangent vector $t$, the estimate for the next solution is given by

$$
\left[\begin{array}{c}
\theta^{e} \\
V^{e} \\
\lambda^{e} \\
\alpha^{e}
\end{array}\right]=\left[\begin{array}{c}
\theta_{j} \\
V_{j} \\
\lambda_{j} \\
\alpha_{j}
\end{array}\right]+\sigma\left[\begin{array}{c}
d \theta \\
d V \\
d \lambda \\
d \alpha
\end{array}\right]
$$


where the superscript " $e$ " stands for estimate, that is, the tangent vector is used to obtain an estimate for $\theta, V, \lambda$ and $\alpha$ starting from the current solution $j$. The symbol $\sigma$ is a scalar that defines the predictor step size. Its value must be such that the estimate is within the radius of convergence of the corrector step.

The secant method uses the current and the previous solutions in order to estimate the next one. Therefore, this is an approximation of tangent predictor. The main advantage of this predictor is that it is relatively inexpensive in term of cpu time and has no problems related to the singularity of the Jacobian matrices. However, the tangent predictor is usually more accurate than the secant predictor, while it cannot foresee control limit effects any better than the secant.

A trivial predictor is the modified zero-order polynomial $[4,8]$, which uses the current solution and a fixed increment in the parameter $\left(V_{k}, \theta_{k}, \lambda\right.$ or $\left.\alpha\right)$ as an estimate for the next solution. Note that the repeated conventional power flow approach corresponds to a continuation method with a modified zero-order predictor.

\subsection{Parameterization and Corrector Step}

Finally, the corrector step solves the case from the estimated solution until convergence of the power flow. After the prediction has been made, it is necessary to correct the approximate solution to avoid error accumulation. Since the point obtained by a good predictor is very close to the correct solution, few iterations will be performed to obtain an exact solution. Newton's method is used in the corrector step. In this step, equation $\alpha-\alpha^{e}=0$, where $\alpha$ and $\alpha^{e}$ are respectively the variable selected as the continuation parameter and its predicted value, is appended to (3.1). So, decomposed the equation (3.1) in Series of Taylor, the correct solution is computed by solving

$$
\left[\begin{array}{cc}
-J & -G_{\lambda} \\
\frac{\partial W}{\partial x} & -\alpha
\end{array}\right]\left[\begin{array}{c}
\Delta \theta \\
\Delta V \\
\Delta \lambda
\end{array}\right]=J_{m}\left[\begin{array}{c}
\Delta \theta \\
\Delta V \\
\Delta \lambda
\end{array}\right]=\left[\begin{array}{c}
\Delta P \\
\Delta Q \\
\Delta W
\end{array}\right]
$$

which is solved by Newton method. For $\alpha^{e}$ the solution of (3.5) gives the new point on the curve $\left(\theta^{2}, V^{2}\right.$ and $\left.\lambda^{2}\right)$ corresponding to the intersection of the solution trajectory curve with the line with new angular coefficient specified by $\alpha^{e}$.

\section{Test Results}

The first point is computed by a conventional Newton method. The convergence criterion adopted to change the step size and parameters during iterative process is a predefined number of iterations associated to the total mismatch criterion. The total mismatch is defined as the sum of the absolute values of the real and reactive power mismatches. The tests are performed considering a convergence threshold of $10^{-4}$ p.u. for total mismatch and a maximum number iterations of ten. 


\subsection{A General Procedure for Changing the Set of Lines}

It was necessary to define a procedure to choose the best set of lines to be used at each stage of the P-V curve. After many tests we concluded that the following procedure is highly robust and also provides a low demand in terms of the total number of iterations needed to build the P-V curves. Its main steps are:

1. solve the base case using a conventional Newton method $(\lambda=1)$ and $\alpha^{1}$ is computed by (3.2), Figure 1;

2. the remaining lines are obtained by applying a step size of 0.02 for $\sigma$ using equation (3.3) predictor and (3.5) corrector, then is calculated for each predictor, a new estimated value of $\alpha$ for the correction until not find more solution;

3. if the PCPF not find solution, it is estimated the coordinates of the set of lines to the median point, between the last two obtained points (points " $a$ " and " $b ")$, i.e., $\left[\left(V_{a}+V_{b}\right) / 2,\left(\lambda_{a}+\lambda_{b}\right) / 2\right]$ will be the coordinates of the second set of lines, called median point $(M P)$, Figure $3(\mathrm{~b})$. The $\alpha^{1}$ is calculated by the line which passes by the coordinates of the center of set of lines (median point) and the last point solved (point " $b ")$.

4. when $V_{k}$ is greater than $V_{k-1}$, it is estimated more 4 points, then, consider the equation of the line that passes by the coordinates initial of set of lines (point " $O$ ") and the last point solved and complete the trace of the P-V curve with $\sigma=0.02$

\subsection{Performance of the PCPF for the IEEE-118 Bus System}

The Figure 2 illustrates the results of the PCPF for tracing P-V curves of the IEEE-118 bus system. Figure $2(\mathrm{a})$ presents the voltage at the critical bus $13\left(V_{13}\right)$ as function of $\lambda$ (P-V curve), with the $\alpha$ as parameter, along with the lines used for solving each point. Point $P\left(\lambda^{1}=1.0 ; V^{1}=0.9680 p . u\right.$. $)$ was solved by a conventional $\mathrm{PF}$. The angular coefficient of the first line $\left(\alpha^{1}=0.2680\right)$ considering that the initial point is the origin $(0,0.7)$, was computed by using equation (3.2). In the Figure 2(b) can be seen the points obtained along the P-V curve of the bus 9 , for the parameter $\alpha$ of the Figure 2(a). The number of iterations performed for solving each point with the PCPF and the CPF with $V_{13}$ as parameter can be seen in the Figure 2(c), where after the point 27, the CPF with $V_{13}$ as a parameter not will pass because of the singularity of the matrix $J$, i.e., will return of the same way of solution already obtained. From these results it can be concluded that the PCPF have a good performance along the solution trajectory even in the vicinity of the MLP and beyond, providing solutions in the lower part of the curve keeping a reduced number of iterations. It should be emphasized that the MLP, and so the loading margin (LM), is obtained after the solution of few points on the curve.

Figure 3 shows similar results of the bus 13. Figure 3(a) presents the P-V curve of the bus (PQ) $44\left(V_{44}\right)$ as function of $\lambda$ (P-V curve), with the $\alpha$ as parameter. It is observed that when the PCPF not find solution for the first set of lines, it is 


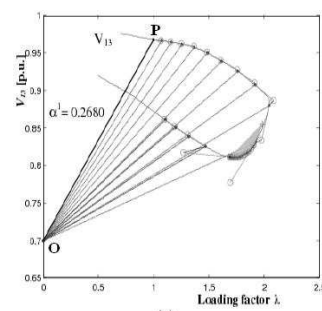

(a)

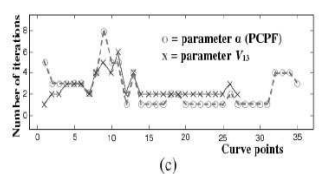

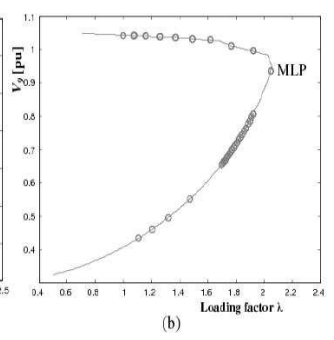

Figure 2: System IEEE-118: (a) Plane $\lambda-V_{13}$ with $\alpha$ as parameter, (b) points of the curve P-V obtained, (c) number of iterations.

estimated the coordinates of the second set of lines to the median point between the last two points obtained (points "a" and " $b$ "), and after the solution of few points on the curve changes to the step 4 of the general procedure and complete the trace of the P-V curve. The Figure 3(c) shows the critical bus with the points obtained through of the bus 44 . The number of iterations performed for getting each point can be seen in Figure 3(d). If it were used the CPF parameterized by $V_{44}$ or $\lambda$ to the obtaining of P-V curve of the bus 44 , the method would be restricted only in the upper part of the curve and often without really knowing if the value is the MLP. It would be need an exchange of parameters to eliminate the singularity of the matrix $J$ and get the MLP. This occurs because the "noses" of the curves are coincident. The same occurs for the bus 46 of Figure 4 to use $V_{46}$ or $\lambda$ as parameter.
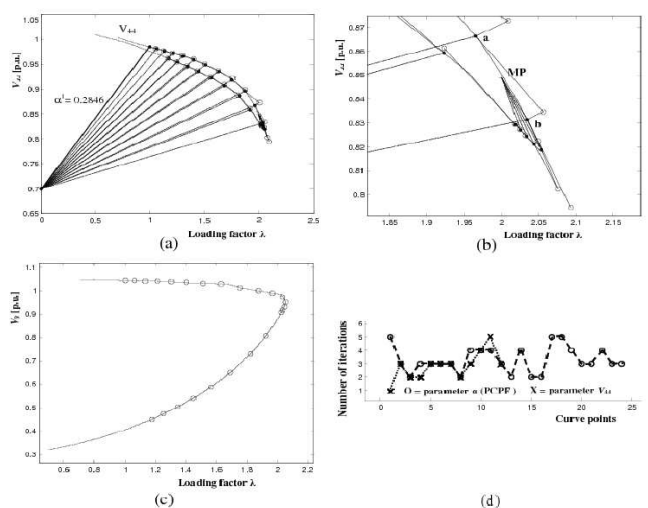

Figure 3: System IEEE-118: (a) Plane $\lambda-V_{44}$ with $\alpha$ as parameter, (b) detail of the MLP region, (c) points of the curve P-V of the bus $V_{9}$ obtained, (d) number of iterations. 

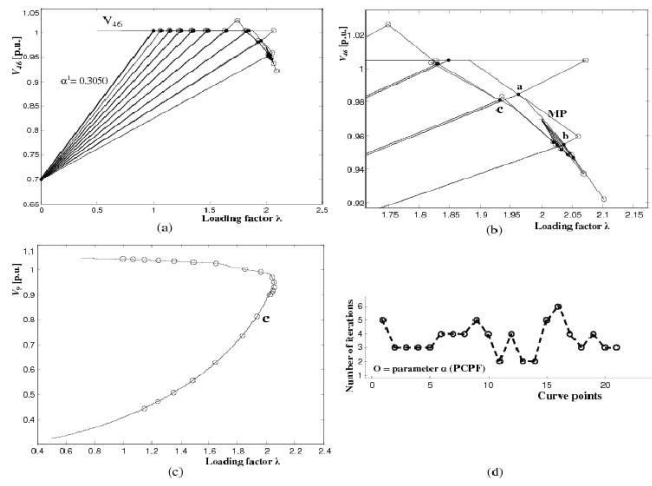

Figure 4: System IEEE-118: (a) Plane $\lambda-V_{44}$ with $\alpha$ as parameter, (b) detail of the MLP region, (c) points of the P-V curve of the bus $V_{9}$ obtained, (d) number of iterations.

Consider now the P-V curve of a bus 46 (PV), figure 4, whose magnitude voltage remains constant over a relatively large stretch of the $\mathrm{P}-\mathrm{V}$ curve, in the case of the generation bus of number 46, see Figure 4 . The Figure 4(a) presents the voltage at the bus $46\left(V_{46}\right)$ as function of $\lambda$ (P-V curve), with the $\alpha$ as parameter, along with the lines used for solving each point. Point " $P$ " was solved by a conventional PF. The angular coefficient of the first line $\left(\alpha^{1}=0.3050\right)$ considering that the initial point is the origin $(0,0.7)$, was computed by using equation $(3.2)$. The other lines were obtained using the equations (3.3), (3.4) and (3.5). There was the exchange of set of lines (i.e., to the median point) with step of 0.2 for $\sigma$, Figure 4 (b), where the singularity of the matrix $J$ was eliminated. Note that the PCPF get success in tracing of the entire $\mathrm{P}-\mathrm{V}$ curve, including the part constant in the curve and the lower part of the curve, Figure 4(a) and (b). In the Figure 4(c) can be seen the points obtained along the $\mathrm{P}-\mathrm{V}$ curve of the bus 9 , for the parameter $\alpha$ of the figure 4(a). The objective is to show that the points obtained actually belong to the upper and lower part of the $\mathrm{P}-\mathrm{V}$ curve, i.e., it is possible to obtain the $\mathrm{P}-\mathrm{V}$ curve complete without the exchange of parameter, this process is verified by point " $c$ " of Figure 4(b) and (c). The number of iterations spend for solving each point with the PCPF can be seen in figure $4(d)$.

\section{Conclusion}

This paper presents a new scheme for the parameterization step of continuation methods aiming the complete tracing of $\mathrm{P}-\mathrm{V}$ curves without the exchange of parameter. The proposed methodology allows the successful computation of any point on the $\mathrm{P}-\mathrm{V}$ curve, with the desired precision, and keeping low requirements in terms of the number of iterations of the convergence process. Another important aspect to be pointed out is that the results obtained for many systems agree with other methods published before [2]. A procedure to switch from a set of lines to other, during the tracing of the $\mathrm{P}-\mathrm{V}$ curves is also presented, if necessary. The automatic 
switching process is done with the purpose of avoiding singularity points on the Jacobian matrix, and also to keep a low requirement in terms of the number of iterations. Even though sometimes it is necessary to change from one set of line to another, the proposed method has as advantage that, unlike other methods, the next set of lines is known in advance. Besides, the change of set of lines does not modify the Jacobian element position but only their values. It can be concluded from many tests performed, that the method represents a very attractive option for building P-V curves and computing the MLP of power systems. It is also of very easy computational implementation, given that few modifications on the conventional power flow program would be required. It also showed that the use of this technique enlarges the group of voltage variables that can be used to obtain the P-V curves.

Resumo. Este trabalho apresenta um novo esquema de parametrização geométrica para o fluxo de carga continuado que possibilita o traçado completo das curvas $\mathrm{P}-\mathrm{V}$, e o cálculo do ponto de máximo carregamento de sistemas de potência sem a troca de parâmetro. Foi implementado o preditor tangente, então para resolver o problema de singularidade da matriz Jacobiana foi adicionado a equação de uma reta que passa por um ponto no plano formado nas variáveis fator de carregamento e magnitude das tensões nodais. O uso desta técnica amplia o grupo das variáveis de tensão que podem ser usadas para o traçado das curvas $\mathrm{P}-\mathrm{V}$. Os resultados obtidos para o sistema IEEE 118 barras mostram que as características do método convencional são melhoradas e a região de convergência ao redor da singularidade é ampliada.

Palavras Chaves. Fluxo de Carga, Multipla Soluções, Ponto de Máximo Carregamento.

\section{References}

[1] V. Ajjarapu, C. Christy, The continuation power flow: a tool for steady state voltage stability analysis, IEEE Trans. on Power Systems PS-7 (1992), 416423.

[2] D.A. Alves, L.C.P. da Silva, C.A. Castro, V.F. da Costa, Continuation load flow method parameterized by power losses, in "Proc. IEEE Power Engineering Society Winter Meeting", pp. 1123-1128, 2000.

[3] D.A. Alves, L.C.P. da Silva, C.A. Castro, V.F. da Costa, Parameterized fast decoupled power flow methods for obtaining the maximum loading point of power systems - part-I: mathematical modeling, Electric Power Systems Research, 69 (2004), 93-104.

[4] C.A. Cañizares, F.L. Alvarado, C.L. DeMarco, I. Dobson, W.F. Long, Point of collapse methods applied to ac/dc power systems, IEEE Trans. on Power Systems, PS-7 (1992), 673-683. 
[5] H.D. Chiang, A.J. Flueck, K.S. Shah, N. Balu, CPFLOW: a practical tool for tracing power systems steady-state stationary behaviour due to load and generation variations, IEEE Trans. on Power Systems, PS-10 (1995), 623-633.

[6] K. Iba, H. Suzuki, M. Egawa, T. Watanabe, Calculation of critical loading condition with nose curve using homotopy continuation method, IEEE Trans. on Power Systems, PS-6 (1991), 585-593.

[7] Y. Mansour, "Suggested Techniques for Voltage Stability Analysis", IEEE Power Engineering Subcommittee Report, 93TH0620-5-PWR, 1993.

[8] T.J. Overbye, R.P. Klump, Effective calculation of power systems low-voltage solutions, IEEE Trans. on Power Systems, PS-11 (1996), 75-82.

[9] Reactive Power Reserve Work Group, "Final Report: Voltage Stability Criteria, Undervoltage Load Shedding Strategy, and Reactive Power Reserve Monitoring Methodology", 1999.

[10] R. Seydel, "From Equilibrium to Chaos: Practical Bifurcation and Stability Analysis", Springer - Verlag, New York, 2th edn., 1994. 